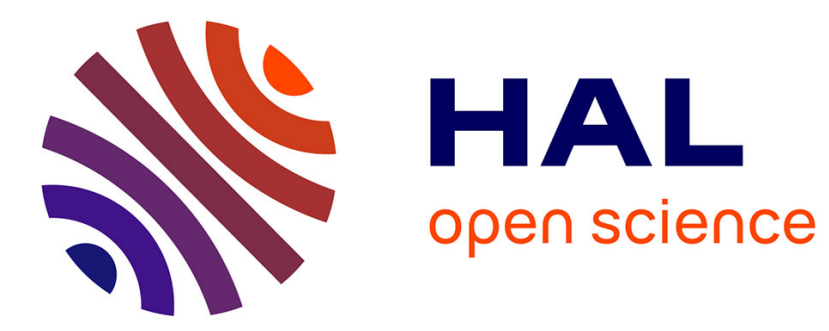

\title{
Digital vibes : la numérisation de Vibrations
}

Jedediah Sklower

\section{To cite this version:}

Jedediah Sklower. Digital vibes: la numérisation de Vibrations. Volume! La revue des musiques populaires, 2018, 14 (2), pp.243-245. 10.4000/volume.5666 . hal-02189893

\section{HAL Id: hal-02189893 \\ https://hal.science/hal-02189893}

Submitted on 13 Nov 2020

HAL is a multi-disciplinary open access archive for the deposit and dissemination of scientific research documents, whether they are published or not. The documents may come from teaching and research institutions in France or abroad, or from public or private research centers.
L'archive ouverte pluridisciplinaire HAL, est destinée au dépôt et à la diffusion de documents scientifiques de niveau recherche, publiés ou non, émanant des établissements d'enseignement et de recherche français ou étrangers, des laboratoires publics ou privés. 


\section{Tribune}

\section{Digital vibes : la numérisation de Vibrations}

\section{Jedediah Sklower (université Paris 3)}

À l'automne 20I3, l'équipe de Volume! était en train de conclure l'édition du numéro «Écoutes » (n० 10-1), dont j'assurais la direction. L'idée de numériser la collection complète de Vibrations m'est venue alors que j'échangeais avec Antoine Hennion, pendant la mise en forme de la version française de son article «La production du succès. Une anti-musicologie de la chanson de variétés " (2013), d'abord publié en anglais dans l'un des tout premiers numéros de la revue Popular Music (en 1983), et constitué de paragraphes tirés de différents chapitres de son ouvrage Les Professionnels du disque : une sociologie des variétés (1981). Nous étions alors engagés dans un aller-retour d'emails pour en retrouver et souder les différents paragraphes. J'avais lu plusieurs articles de Vibrations, notamment ceux de Jean-Claude Klein et Pascal Ory $\left(n^{\circ} 1\right)$ à l'époque de mon D.E.A. sur l'histoire du free jazz en France, et découvert grâce à l'équipe de Volume!l'ouvrage pionnier Rock, de l'histoire au mythe, dont je m'étais servi pour préparer un cours d'histoire et d'esthétique des musiques populaires - notamment le fameux «Mais pourquoi donc en 1955 ? » de
Richard Peterson, traduit... par Antoine Hennion, ainsi que ceux de Philippe Teillet sur la politique culturelle du rock en France, de Patrick Mignon sur la scène de Givors, d'Érik Neveu sur l'idéologie. J'avais fait photocopier celui de Peterson la première année à Lille où je donnais ces cours, mais je désespérais d'avoir une version numérique, pour m'épargner la paperasse et le recours au service reprographie de la faculté. Le travail sur le numéro "Écoutes », démarré plus de trois ans plus tôt, arrivait à sa conclusion, et nous cherchions de nouvelles idées pour continuer à faire prospérer la vocation de l'association Mélanie Seteun.

Celle de diffuser une version numérique de Vibrations et de Rock, de l'histoire au mythe 1 s'imposa comme une évidence. Une revue et un ouvrage pionniers de l'étude en France des musiques populaires, représentative, comme le rappelle Antoine Hennion dans son texte, d'une certaine conjoncture intellectuelle dans les sciences sociales - la lutte contre l'hégémonie de la sociologie bourdieusienne
1 D'après les souvenirs d'Antoine Hennion, il s'agissait avec Rock, de l'histoire au mythe de développer une collection indépendante d'ouvrages, lancée par Comment la musique vient aux enfants (Hennion, 1990), publié chez Economica (coll. Anthropos). Pourtant, s'il ne fut pas publié chez Privat, qui décida d'abandonner la collection, il portait néanmoins fièrement sur sa couverture le sceau "Collection Vibrations ", ainsi qu'une référence au titre complet de la revue en page de titre. Par ailleurs, étaient annoncés dès le $n^{\circ} 4$ de la revue (1987) un dossier " rock " (censé être le $n^{\circ} 8$, suivant les six premiers ainsi qu'un septième intitulé « Mélanges : ouvertures et débats "suscités par les 4 premiers numéros), de même qu'un hors-série “ La Révolution française " à l'occasion du bicentenaire, qui donnera l'ouvrage Orphée phrygien. Les musiques de la révolution (Klein \& Julien, 1989), publié quant à lui aux éditions du May, mais toujours sous le patronage de la " revue Vibrations ", en surtitre sur la couverture. 
de la culture (Hennion, Neveu, etc.), les premiers développements de l'histoire culturelle des musiques populaires en France (Klein, Philippe Gumplowicz, Ory, etc.), l'ouverture de disciplines anciennes (la linguistique, avec Louis-Jean Calvet, la musicologie, avec Julien) et nouvelles (les sciences de l'information et de la communication, avec François Jost) aux musiques populaires, mais avant l'importation des différentes déclinaisons des «studies». Une revue dont l'audace avait inspiré mes camarades de Volume!, le modèle inédit à nos yeux d'un certain tissage de liens entre des démarches et des disciplines, dont nous voulions perpétuer l'esprit au sein de notre travail associatif.

Je soumis le projet à Antoine Hennion, son directeur de la publication, qui l'accueillit avec beaucoup d'enthousiasme. Avec son aide, je commençai aussitôt par contacter les épouses des deux autres fondateurs, le musicologue Jean-Rémy Julien et l'historien Jean-Claude Klein ${ }^{2}$, ainsi que le codirecteur de Rock, de l'histoire au mythe, Patrick Mignon, tous trois des tisseurs-passeurs passionnés de nombreuses facettes des cultures populaires. Je me mis ensuite en relation avec les maisons d'édition qui nous accordèrent, nonchalants et bienveillants à la fois, les droits pour les versions numériques, gratuitement. Comme me le rappela Élisabeth Knebelmann des Éditions Privat, étant donné que la revue était épuisée et que la maison n'avait pas entrepris de la rééditer, les textes ne lui appartenaient de toute façon plus ${ }^{3}$. Puis je proposai l'idée

2 Seule Catherine Goussef Klein m'a répondu; je n'ai pas réussi à joindre Marion Julien. à nos partenaires d'OpenEdition, qui me redirigèrent vers Persée, plateforme plus spécifiquement consacrée à la réédition numérique de collections complètes de revues, notamment les plus anciennes, et avec lesquels je pris contact à l'automne 2014 lors du Salon de la Revue, où notre revue tient un stand depuis plusieurs années. Que tous soient ici remerciés pour leur soutien inconditionnel à ce projet, qui nous a permis, dans ces circonstances, de rester fidèles à nos principes de diffusion ouverte de la recherche française sur les musiques populaires.

Émilie Paget et Philippe Gissinger, mes interlocuteurs du côté de Persée, m'expliquèrent les étapes du processus : constitution d'un dossier de partenariat (validé en avril 20I5), contact des auteurs ou de leurs ayants droit, signature d'une convention tripartite Persée-Volume!-Vibrations (représentée par A. Hennion), envoi d'une collection complète pour massicotage et numérisation, constitution des métadonnées, validation éditoriale des contenus puis enfin mise en ligne. Il fallait notamment recueillir l'accord de suffisamment de contributeurs pour que l'ensemble puisse être publié sans risquer, si beaucoup d'entre eux optaient pour le retrait de leurs textes ${ }^{4}$, de se retrouver avec des numéros en haillons. Je reconstituai donc la liste des quelques II5 auteurs d'articles de recherche, de notes de lecture, d'entretiens, de comptes-rendus de conférences, de notes

4 Nous aurions très bien pu décider de ne mettre en ligne que les textes pour lesquels nous avions obtenu ces autorisations, et poursuivre l'édition à partir d'un principe d'"opt-in ". Mais nous étions confiants que personne ne s'offusquerait de ce choix, connaissant un peu l'esprit de chercheurs travaillant sur des objets longtemps discrédités, ainsi que celui qui avait présidé au travail au sein de la revue. 
diverses (événements, associations, publications...), de professionnels, de techniciens, de musiciens et d'autres ayant publié dans la revue, et me mis en quête de moyens de les contacter. Une aventure quasi impossible avant l'ère du numérique. Les résultats de cette pêche furent très fructueux : toutes les personnes m'ayant répondu ( $43 \%$ des auteurs, représentant deux tiers des textes de la revue) soutinrent le projet avec joie. Ce fut l'occasion pour certains d'entre eux de reprendre contact, de se remémorer une époque, l'esprit d'une revue, un violon d'Ingres peut-être abandonné depuis.

Il est à cet égard assez étonnant qu'il n'y ait eu qu'une contributrice pour m'interroger sur la politique de la plateforme en matière de publication - elle craignait une privatisation lucrative des textes, démarche contraire aussi bien à l'esprit de Volume! qu'aux règles de Persée. La question de la numérisation de la production scientifique française (et mondiale) pose évidemment des enjeux cruciaux à l'ère de l'émulation quantophrénique par le "publish orperish", de la "gouvernance algorithmique », du « digital labor», de la mesure statistique de soi et des récentes injonctions au libre accès immédiat. Le fait qu'il s'agît de textes quasi trentenaires, publiés avec l'aval du directeur de publication, par une association, sur le portail d'une unité mixte de service associant l'université de Lyon, l'ENS Lyon et le CNRS, suffit probablement à désamorcer des réticences légitimes.

La dernière étape fut celle de la " validation éditoriale » que je lançai en février 20 I6 avec l'aide de Thomas Mansier : il s'agit essentiellement de vérifier les droits sur les illustrations, la table des matières des numéros, le plan des articles, les titres et sous-titres, de corriger les coquilles ou les données trop « littéralement » consignées (notamment les titres d'illustrations, souvent très longs), pour que la trame présentée aux lecteurs soit fidèle à la version écrite et fluide à la navigation. Les premières « autorisations de diffusion » furent accordées aux deux premiers numéros de Vibrations et à Rock, de l'histoire au mythe; les numéros 3 et 4 suivirent à l'automne 20I6, et l'ensemble fut complété en février 2017 - plus de trois ans, tout de même... Le travail doit continuer! Que l'on pense aux autres ouvrages publiés par l'équipe ou à une revue comme Musique enjeu, par exemple. Avis aux passionnés!

\section{Bibiographie}

Hennion Antoine (2013), « La production du succès : une antimusicologie de la chanson de variétés ", Volume! la revue des musiques populaires, vol. $9, \mathrm{n}^{\circ} \mathbf{1}$, p. 47-73, en ligne : https://volume. revues.org/3815.

\author{
- (1990), Comment la musique vient \\ aux enfants. Une anthropologie \\ de l'enseignement musical, Paris, \\ Economica. \\ - (1981), Les Professionnels du \\ disque. Une sociologie des variétés, \\ Paris, Métailié.
}

Klein Jean-Claude \& Julien Jean-Rémy (eds.) (1989), Orphée phrygien. Les musiques de la révolution, Paris, du May. 